\title{
Tissue-specific epigenetics of atherosclerosis-related ANGPT and ANGPTL genes
}

\author{
Kenneth C Ehrlich', Michelle Lacey ${ }^{2,3}$ \& Melanie Ehrlich*,1,3,4 \\ ${ }^{1}$ Center for Bioinformatics \& Genomics, Tulane University Health Sciences Center, New Orleans, LA 70112, USA \\ ${ }^{2}$ Department of Mathematics, Tulane University, New Orleans, LA 70118, USA \\ ${ }^{3}$ Tulane Cancer Center, Tulane University Health Sciences Center, New Orleans, LA 70112, USA \\ ${ }^{4}$ Hayward Genetics Center Tulane University Health Sciences Center, New Orleans, LA 70112, USA \\ *Author for correspondence: Tel.: +1 504988 2449; ehrlich@tulane.edu
}

\begin{abstract}
Aim: To understand tissue-specific regulation of angiopoietin/angiopoietin-like (ANGPT/ANGPTL) genes (especially the five genes embedded in introns of host genes) and their association with atherosclerosis. Methods: Transcription and epigenomic databases from various normal tissues were examined in the vicinity of ANGPT1, ANGPT2, ANGPTL1, ANGPTL2, ANGPTL3, ANGPTL4 and ANGPTL8. Results: We identified tissue-specific enhancer chromatin regions that are likely to regulate transcription of $A N G P T / A N G P T L$ genes and were intragenic, intergenic or host gene-linked. In addition, we found atherosclerosis-linked differentially methylated regions associated with ANGPT2 and with sequences encoding miR-145, a microRNA that targets ANGPT2 mRNA in cancers. Conclusion: Our findings implicate enhancers as major contributors to tissue-specific expression of ANGPT/ANGPTL genes, which play critical roles in angiogenesis, atherosclerosis, cancer, and inflammatory and metabolic diseases.
\end{abstract}

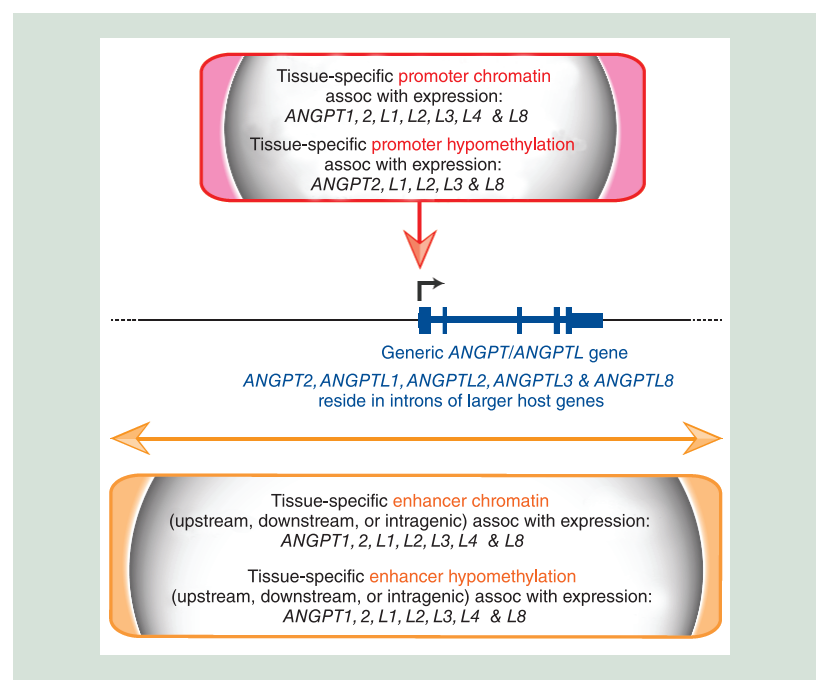

First draft submitted: 14 September 2018; Accepted for publication: 23 October 2018; Published online: 28 January 2019

Keywords: angiogenesis $\bullet$ ANGPT2 $\bullet$ ANGPTL3 $\bullet$ ANGPTL4 $\bullet$ ANGPTL8 $\bullet$ atherosclerosis $\bullet$ cancer $\bullet$ DNA methylation - enhancers • miR-145

Atherosclerosis is a progressive disease involving damage to the arterial lumen's endothelial cell barrier and accompanying leukocyte infiltration, changes in endothelial cells and the subendothelial extracellular matrix and retention of cholesterol-containing low-density lipoprotein (LDL) in the artery wall $[1,2]$. Also critical to pathogenesis are smooth muscle cell $(\mathrm{SMC})$ phenotypic transformations in the arterial wall and intra-aorta migration of SMC [3,4]. Damage 
to the aorta involves loss of elasticity, inflammation, calcification and, eventually, necrosis and apoptosis [5]. Among the proteins implicated in the initiation and progression of atherosclerosis are angiopoietin (ANGPT) proteins, which bind to TIE1 or TIE2 receptor tyrosine kinases, and most of the angiopoietin-like (ANGPTL) proteins, which bind to other receptors and have one or two shared domains not present in ANGPT proteins [6,7].

The clinically important secreted ANGPT/ANGTPL proteins play many roles in development and disease including in angiogenesis, inflammation, lipid and glucose metabolism, hematopoietic stem cell activity and cancer [6,8]. Seven of the eleven $A N G P T / A N G P T L$ genes have been implicated in atherosclerosis, namely, ANGPTL1, ANGPTL2, ANGPTL3, ANGPTL4 (ANG4), ANGPTL8, ANGPT1 (ANG1) and ANGPT2 (ANG2). Their encoded proteins have roles in lipid deposition in the aorta, vascular growth, vessel permeability and remodeling, vascular inflammation, neovascularization and plaque destabilization during disease progression $[6,7,9,10]$. Two of the ANGPT/ANGPTL genes, ANGPTL6 and ANGPTL7 [6], are associated with obesity, a risk factor for atherosclerosis $[11,12]$.

Some of the ANGPT/ANGPTL proteins can be either pro-atherosclerotic or protective against atherosclerosis depending on the stage of the disease and the cellular context [6,10]. Their pro-atherosclerotic activity is due, in part, to favoring chronic inflammation (ANGPTL2) as well as helping to transport lipoproteins into the atherosclerotic or pre-atherosclerotic aorta and inhibiting lipoprotein lipase (LPL) and endothelial lipase (namely, ANGPTL3, ANGPTL4 and ANGPTL8) [13-15]. Inhibition of LPL increases circulating levels of LDL and very LDL. Low expression of ANGPTL3 and ANGPTL8 in individuals with heterozygous inactivating mutations or in atherosclerosis mouse models correlates with a reduced risk of atherosclerosis and lower levels of lipid in the bloodstream $[6,15,16]$. ANGPTL3 and ANGPTL8 bind to each other and act cooperatively in their inhibition of LPL activity, which helps determine susceptibility to atherosclerosis $[13,17,18]$. ANGPT1 and ANGPT2 can act as competing or assisting partners mediating angiogenesis $[7,19]$.

Recent research suggests that atherosclerosis is an epigenetic disease involving changes in chromatin and DNA methylation during disease progression [20-23]. Nonetheless, there are only a few reports describing chromatin modifications or DNA methylation at the ANGPT/ANGPTL genes other than the many studies about carcinogenesis-related epigenetic changes in these genes $[6,24,25]$. A complication in the regulation of transcription of ANGPT/ANGPTL family genes, including ANGPTL3 and ANGPTL8, is that many of them are small genes residing in an intron of a large host gene. Understanding how epigenetic changes contribute to the control of gene expression to help maintain precise levels of the corresponding ANGPT/ANGPTL proteins is important not only to atherosclerosis, but also to apoptosis, metabolic diseases, retinopathies, sepsis, cancer and inflammatory diseases like rheumatoid arthritis and dermatomyositis [6,7].

Here, we describe our analysis of whole-genome epigenetic and transcriptomic databases to elucidate epigenetic factors that influence the tissue-specificity of expression of the seven atherosclerosis-associated ANGPT/ANGPTL genes. For this study, we also utilized whole genome-bisulfite sequencing profiles of atherosclerotic and control aorta to look for significantly differentially methylated regions (DMRs) in these genes that are associated with this disease. We also examined the gene region that encodes miR-145, a microRNA (miRNA) that has been shown to target ANGPT2 in cancer [26] and that has been demonstrated to regulate the cellular phenotype of aorta SMCs [27]. Our findings underscore the importance of analyzing the involvement of intragenic, intergenic and host gene-embedded enhancers in controlling expression of these clinically important genes.

\section{Materials \& methods \\ Bioinformatics}

Epigenetic and RNA-seq profiles from the Roadmap and ENCODE projects [28,29] were analyzed using the UCSC Genome Browser or its hubs for bisulfite sequencing (bisulfite-seq) and chromatin state segmentation (chromHMM, AuxilliaryHMM) [30]. Reduced representation bisulfite sequencing (RRBS) methylation profiling [31] was used in analysis of ANGPTL8. The studied tissues were histologically normal adult samples specified in the figures, including brain (prefrontal cortex), muscle (psoas), heart (left ventricle). Cell cultures were cell strains except for the lymphoblastoid cell line (LCL, GM12878) and the cancer cell lines (HepG2, K562, MCF-7 and LNCaP). The color code for the 18-state chromatin state segmentation was slightly simplified from the original [28]. Enhancer chromatin in this study denotes strong enhancer chromatin with states of 3 or 7-10 [28]. Quantification of RNA-seq for tissues was from the GTEx database [32] using median values for reads per kilobase million (RPKM, in figures) or transcripts per kilobase million (in Supplementary Table 1) from many samples for each tissue type. The RNA-seq data track showing alignment of the signal with exons were from the UCSC Genome Browser (Burge tracks, 
vertical viewing range 0-1) [33]. RNA-seq data for cell cultures was from ENCODE UCSC Genome Browser tracks as follows: transcription levels by Long RNA-seq for poly $(\mathrm{A})^{+}$whole-cell RNA with strand-specific analysis on $>200 \mathrm{nt}$ poly $(\mathrm{A})^{+}$RNA (vertical viewing range 0-30 unless otherwise indicated) and Small RNA-seq from total RNA that was $<200$ nt (contigs) from Cold Spring Harbor Laboratories [29]. RNA Subcellular CAGE Localization were from RIKEN Omics Science Center [29].

\section{Determination of atherosclerosis-associated DMRs}

For the atherosclerotic and control aorta samples (plaque and underlying aorta from an 88-year-old female with grade VII atherosclerosis and nonatherosclerotic [Ctl] aorta from that individual), the bisulfite-seq data from Zaina et al. [20] were used. These data were supplemented with two additional control aorta bisulfite-seq profiles from a 34-year-old male and a 30-year-old female (Roadmap Epigenetics Project [28]). Bisulfite-seq data from the atherosclerotic aorta (Athero A) and control aorta (Ctl A) samples were initially merged and analyzed on a site-bysite basis by applying Fisher's exact test to the counts of methylated and unmethylated CpG reads in each sample to produce site-specific p-values, $\mathrm{p}_{\mathrm{i}}$. Based on these results, candidate DMRs were then identified by determining the joint probability of a sequence of five or more consecutive $p$-values $\left(p_{i}, p_{i}+1, \ldots, p_{i}+k\right)$ according to the uniform product (UP) distribution as described in our previous study [34], where each candidate DMR was required to begin and end with a statistically significant site. This analysis identified statistically significant regions at the 0.05 level, and these were subsequently filtered to include only those regions with an average percent methylation difference of at least $\pm 20 \%$, length greater than 250 bp and containing no gaps between consecutive sites greater than 200 bp. Next, these samples were merged with the two additional control aorta samples [28], using custom scripts to correct for single-bp shifts due to variable pre-processing routines. For all sites present in all four samples, logistic regression models were fit to the counts of methylated and unmethylated reads at each CpG site to determine the statistical significance associated with the difference in percent methylation between the atherosclerotic aorta (A) and the three controls. Associated p-values for the comparison of athero aorta versus three control aortas were then analyzed using our UP method to identify candidate DMRs, which were subsequently filtered as above for length, percent methylation difference and gaps. Many atherosclerosclerosis-associated DMRs that we determined from these four samples had been validated in a previous study of 15 pairs of atherosclerotic and control aortas subject to a more limited DNA methylation analysis [20,35].

\section{Statistical analyses of expression data}

Expression profiles across 38 tissues (GTEx, Supplementary Table 1) [32] for selected genes were analyzed to evaluate differences in median RNA levels with respect to liver expression using nonparametric Wilcoxon rank tests. To robustly assess associations in expression profiles between pairs of genes, tests using both Pearson correlations on the $\log _{2}$ expression values and rank-based Spearman correlations were employed.

\section{Results}

Tissue-specificity of expression of genes in the ANGPT \& ANGPTL families

We first used the GTEx database [32], which contains RNA-seq data from hundreds of individuals for each tissue type, to compare expression of ANGPT and ANGPTL family genes in 51 different kinds of human tissue to confirm and extend our understanding of the genes' tissue-specific expression. By examining steady-state RNA levels rather than protein levels, we can address the tissue-specific generation of these blood-borne proteins without the complication of their distribution through the circulatory system. All the genes in this family exhibit tissuespecific expression (Figure 1), with the highest tissue specificity observed for ANGPTL3 and ANGPTL8 (C19orf80) in liver, $A N G P T L 7$ in tibial nerve and ANGPTL5 in ovary (Supplementary Table 1 and [32]). Not only do ANGPTL8 and $A N G P T L 3$ show a strong preference for expression in liver, but also they are expressed at very high RNA levels in this tissue, as are ANGPTL2 and ANGPTL4 in adipose (Figure 1). ANGPTL3 and ANGPTL8 are transcribed as antisense transcripts from intron 14 of the broadly expressed DOCK7 and DOCK6 genes, respectively (Figures $2 \mathrm{~A}$ $\&$ Figure $3 \mathrm{~A}$ ), which encode guanine nucleotide exchange factors and displayed an inverse relationship between the ANGPTL and host DOCK gene expression in liver. ANGPTL3 and 8 were significantly overexpressed and DOCK 7 and 6 significantly underexpressed relative to the set of 38 other tissues considered (Table 1). The other studied genes-within-genes, which are expressed in multiple tissues, showed either a positive association of their expression with that of the host gene (ANGPT2 / MCPH1, correlation 0.6) or no significant association (ANGPTL1 /RALGPS2 and ANGPTL2 / RALGPS1) in analyses across 39 tissues. 


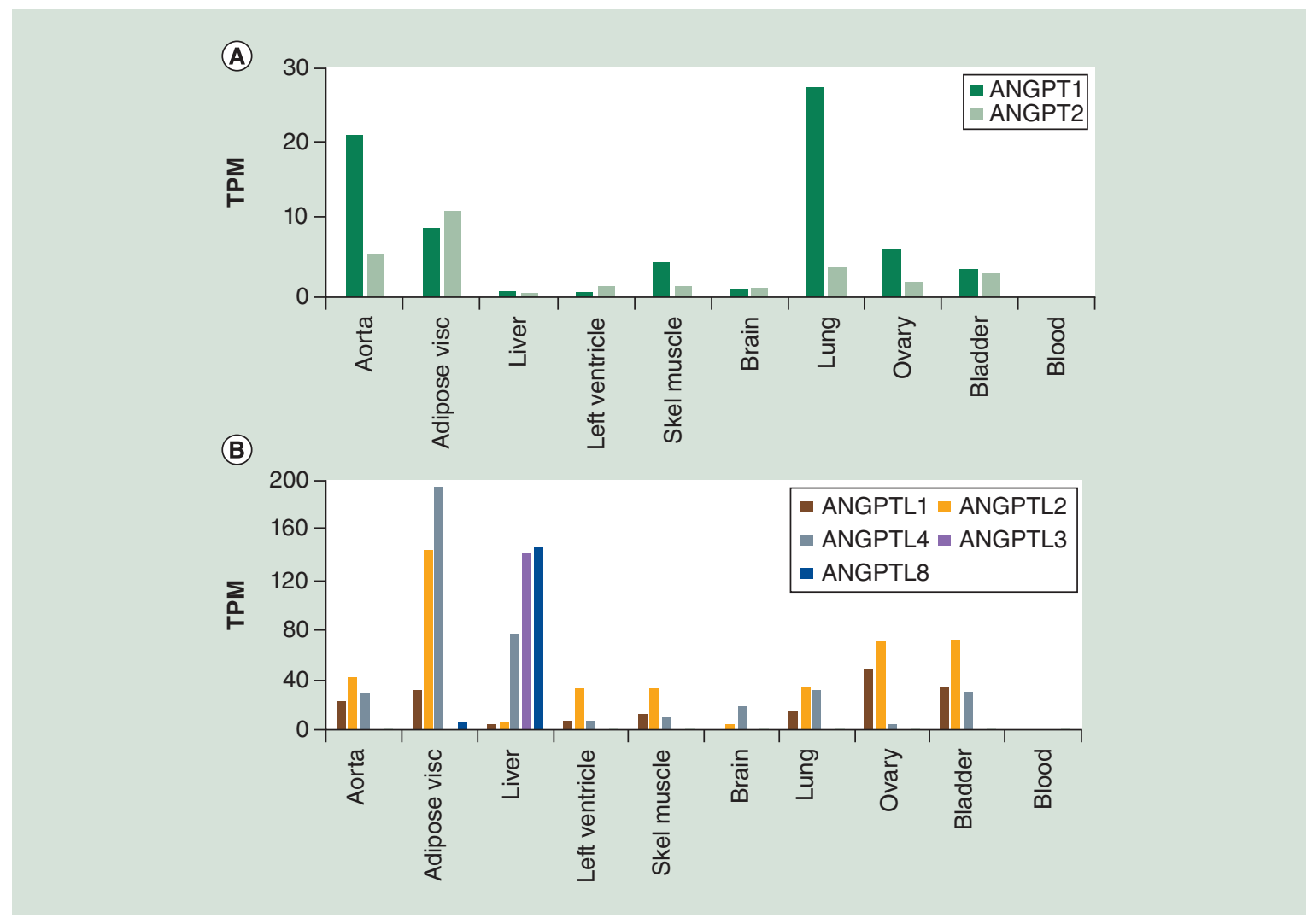

Figure 1. Expression of ANGPT and ANGPTL genes in different tissues. RNA-seq data are from GTEx [32] for ten of the available tissue types and are given as median TPM for ANGPT1 and ANGPT2 (A) and five of the ANGPTL genes (B). Note difference scales of the $y$-axis in (A) and (B). For quantitative data from more tissue types and related genes, see Supplementary Table 1.

Adipose-visc: Adipose from visceral tissue; Skel muscle: Skeletal muscle; TPM: Transcripts per million.

\begin{tabular}{|c|c|c|}
\hline \multirow[t]{2}{*}{ Gene pair } & \multicolumn{2}{|c|}{ Significance of over- and under-expression in liver: $p$-value } \\
\hline & Overexpression & Underexpression \\
\hline 1a. ANGPTL3 & $6.0 \times 10^{-8}$ & NS \\
\hline 1b. DOCK7 & NS & $2.9 \times 10^{-7}$ \\
\hline 2a. ANGPTL8 & $7.9 \times 10^{-7}$ & NS \\
\hline 2b. DOCK6 & NS & $4.3 \times 10^{-7}$ \\
\hline 3b. RALGPS2 & NS & NS \\
\hline 4a. ANGPTL2 & NS & $1.0 \times 10^{-9}$ \\
\hline 4b. RALGPS1 & NS & $8.0 \times 10^{-8}$ \\
\hline 5a. ANGPT2 & NS & $1.4 \times 10^{-7}$ \\
\hline 5b. MCPH1 & NS & $1.0 \times 10^{-7}$ \\
\hline
\end{tabular}

$\dagger$ Expression analysis of five intronic gene/host gene pairs was done by comparing median expression levels for the indicated genes among 39 tissues in the GTEx RNA-seq database. NS: Not significant.

Liver-specific enhancer \& promoter chromatin \& DNA hypomethylation at ANGPTL3

Consistent with its liver-specific expression, ANGPTL3 exhibited liver-specific hypomethylation that overlaps the gene body and the vicinity of the transcription start point (TSS). In liver, strong promoter chromatin (enriched in $\mathrm{H} 3$ lysine- 4 trimethylation, $\mathrm{H} 3 \mathrm{~K} 4 \mathrm{me} 3$, and $\mathrm{H} 3$ lysine-27 acetylation, $\mathrm{H} 3 \mathrm{~K} 27 \mathrm{ac}$ ) extended from $0.9 \mathrm{~kb}$ upstream to $4.5 \mathrm{~kb}$ downstream of the TSS for ANGPTL3 (Figure 2B, dotted box), and, therefore, overlapped almost half 


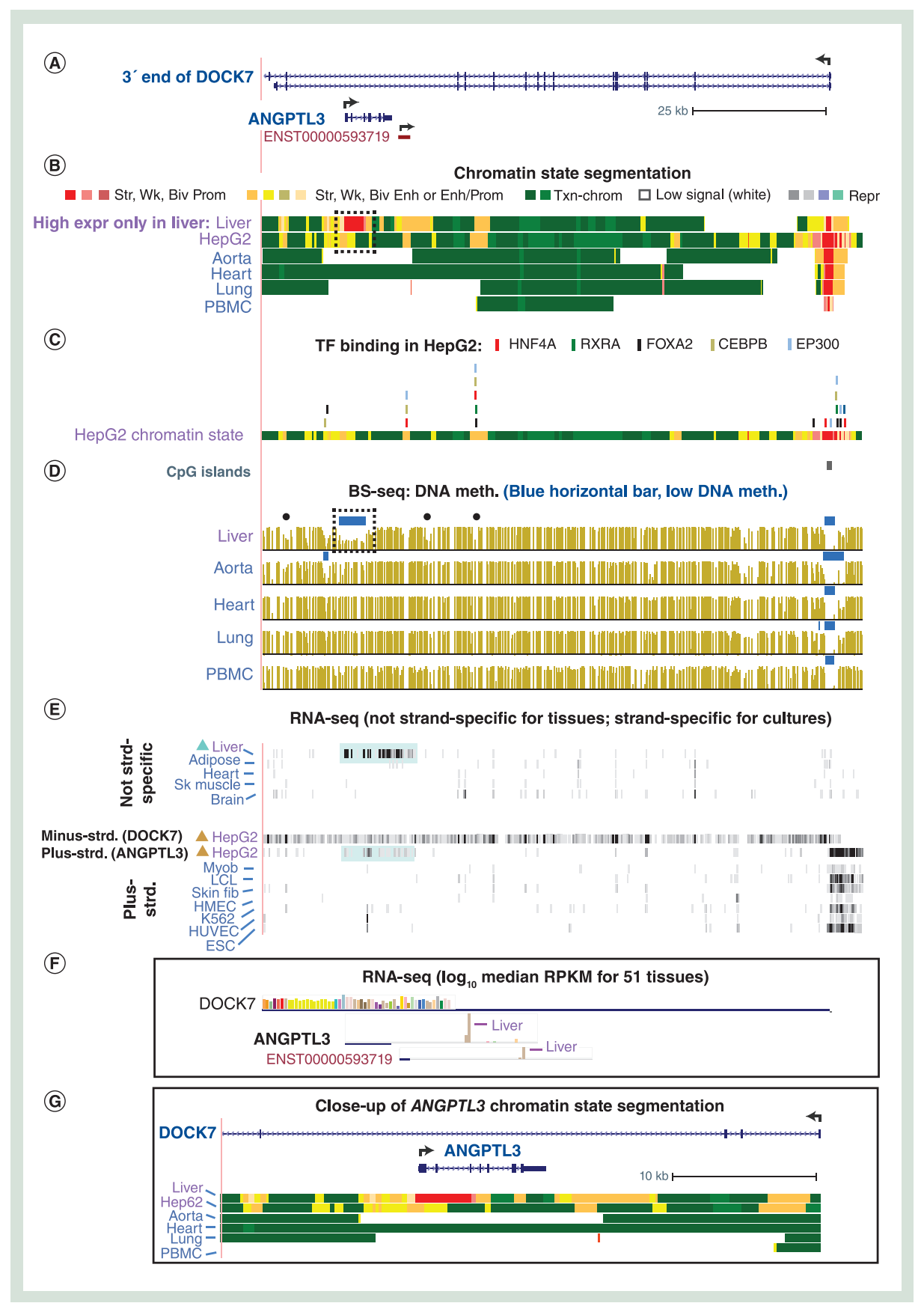

Figure 2. ANGPTL3 displays liver-specific enhancer and promoter chromatin and DNA hypomethylation that correlates with its liver-specific expression. (A) ANGPTL3 resides in an intron of DOCK7, a guanine nucleotide exchange factor-encoding gene (chr1:63,047,768-63,160,178). Blue, RefSeq gene isoforms; dark red, an additional Ensembl transcript-encoding isoform. (B) The color code indicates predicted chromatin states [28]: strong, weak, or bivalent promoter or enhancer; transcribed; repressed (H3K27me3 strong or weak, H3K9me3 or H3K9me3 plus H3K36me3); or low-signal chromatin. (C) TF binding in HepG2 cells as determined by TF ChIP-seq is indicated by the given color code. (D) CpG islands [30] and bisulfite-seq tracks, which are plotted as average \% methylation at CpGs; blue horizontal bars, regions that display statistically significant hypomethylation relative to the rest of the same genome [36]. The dotted boxes indicate the large region exhibiting liver-specific DNA hypomethylation in (D). Black dots indicate additional regions of hypomethylation overlapping enhancer chromatin. (E) RNA-seq data from ENCODE for individual samples $[29,30]$ with the vertical viewing range set at $0-10$ instead of $0-30$ as in subsequent figures. (F) Bar graph of RNA-seq data from multiple samples in the GTEx database [32]; see Supplementary Table 1 for quantitative data. (G) Zoomed-in view of chromatin segmentation tracks from panel (B) showing just ANGPTL3 and the liver-specific enhancer regions around it (chr1:63,049,440-63,091,060). All tracks are aligned and derived from the UCSC Genome Browser with hg19 coordinates in this and subsequent figures.

ESC: Embryonic stem cell; fib: Fibroblast; HepG2: Hepatocarcinoma cell; HMEC: Human mammary epithelial cell; HUVEC: Human umbilical vein endothelial cell; K562: Myelogenous leukemia cells; Myob: Myoblast; PBMC: Peripheral blood mononuclear cells; Sk muscle: Skeletal muscle; TF: Transcription factor. 
(A)

$5^{\prime}$ end of DOCK6

(B)

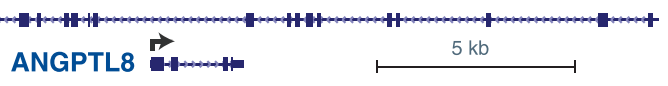

Chromatin state segmentation

$\square \square$ Str, Wk, Biv Prom $\quad \square \square \square$ Str, Wk, Biv Enh or Enh/Prom $\quad \square \quad$ Txn-chrom $\square$ Low signal (white) $\square \square \square \square$ Repr

Very high expr

Moderate expr

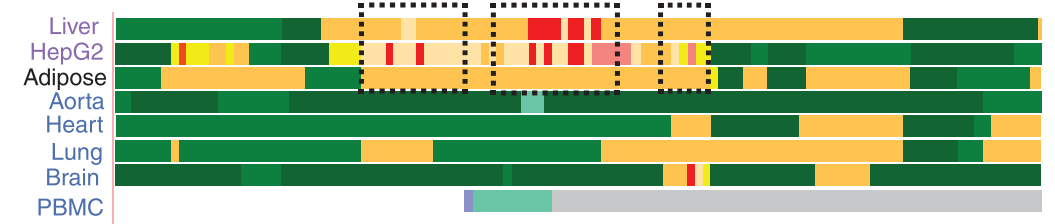

(C)

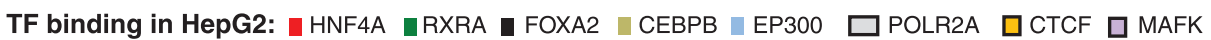

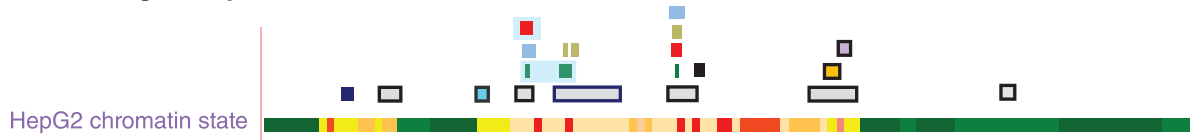

(D)

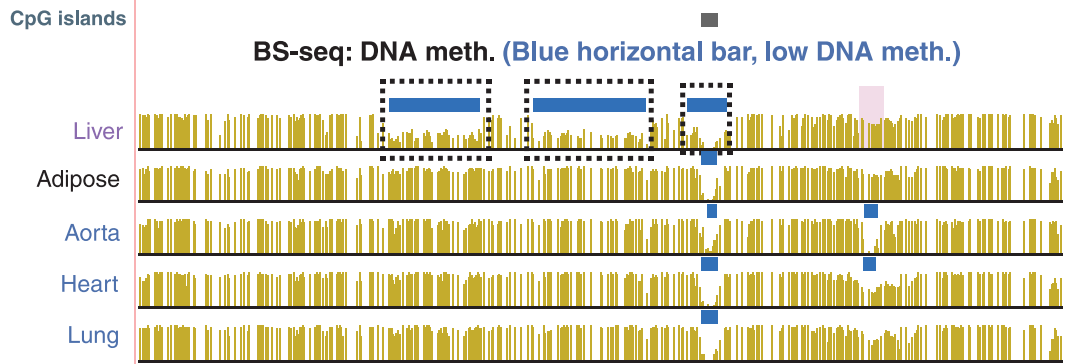

(E)

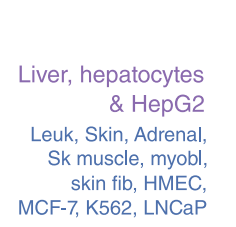

(F)

RRBS: DNA meth. $\square 0 \%$ meth. $\square 50 \%$ meth. $100 \%$ meth.
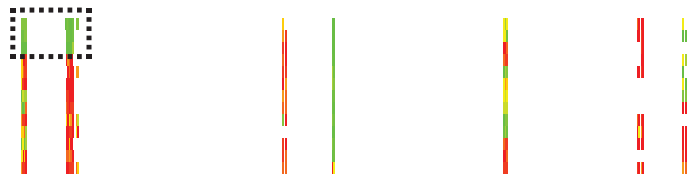

RNA-seq (not strand-specific for tissues; strand-specific for cultures)

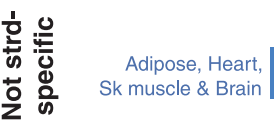

Minus-strd. (DOCK6) HepG2

Plus-strd. (ANGPTL8) HepG2

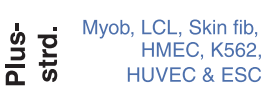
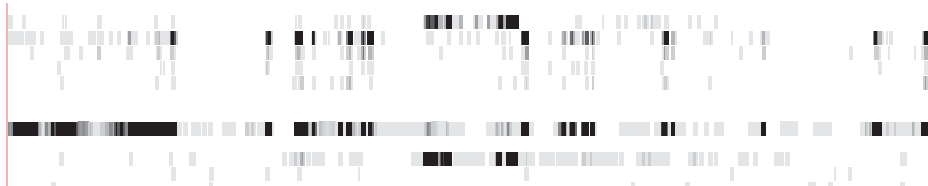

(G)

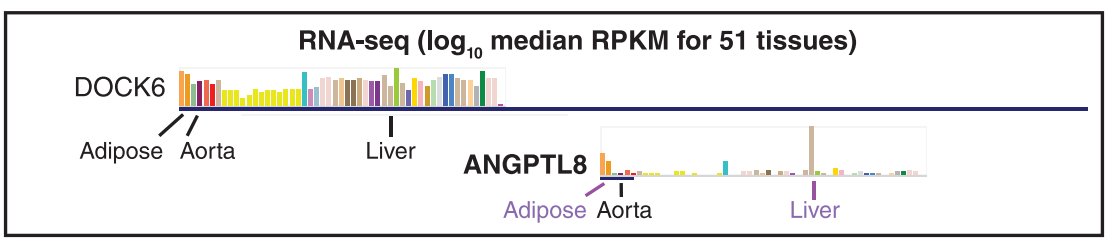

Figure 3. ANGPTL8 exhibits intragenic enhancer chromatin overlapping DNA hypomethylation specifically in liver, which has the highest expression. (A) ANGPTL8 is embedded in intron 14 of 47 introns of DOCK6

(chr19:11,339,832-11,363,081). (B-D) Predicted chromatin states, TF binding sites and DNA methylation profile from bisulfite-seq as in Figure 2. Dotted boxes in (B) and (C) indicate regions where liver-associated DNA hypomethylation was seen. (E) DNA methylation profiling by RRBS (with an 11-color gradient) including cell cultures for which bisulfite-seq DNA are not available. (F) and (G), RNA seq data as in Figure 2. MCF-7 and LNCaP, breast and prostate cancer cell lines, respectively; blue highlighting in panel (C), two neighboring RXRA sites and a HNF4A site that overlaps one of them.

ESC: Embryonic stem cell; Expr: Expression; fib: Fibroblast; HepG2: Hepatocarcinoma cell; HMEC: Human mammary epithelial cell; HUVEC: Human umbilical vein endothelial cell; K562: Myelogenous leukemia cells; LCL: Lymphoblastoid cell line; Myob: Myoblast; PBMC: Peripheral blood mononuclear cells; RRBS: Reduced representation bisulfite sequencing; Sk muscle: Skeletal muscle; TF: Transcription factor. 
of the 8.8-kb gene (Figure 2G). Liver-specific enhancer chromatin (enriched in H3 lysine-4 monomethylation, $\mathrm{H} 3 \mathrm{~K} 4 \mathrm{me} 1$, and $\mathrm{H} 3 \mathrm{~K} 27 \mathrm{ac}$ ) was found upstream of the ANGPTL3 promoter region, immediately downstream of the $3^{\prime}$ end of the gene and $15 \mathrm{~kb}$ downstream of the $3^{\prime}$ end of the gene. These enhancer chromatin regions were all inside the body of the oppositely oriented DOCK7, the host gene for ANGPTL3. Small regions of low methylation overlapped these enhancer chromatin segments specifically in liver (Figure 2D, black dots). At the enhancer chromatin immediately downstream of ANGPTL3 there was an overlapping gene (AL138847.1) aligned with a small single-exon transcript (ENST00000593719) (Figure 2A) [37]. This gene, which was not previously described as being associated with enhancer chromatin, is expressed almost exclusively in liver, like ANGPTL3 (Figure 2F), and therefore might help upregulate ANGPTL3 in liver in cis or trans, like many other ncRNA genes very close to coding genes [38].

HepG2, a hepatocellular carcinoma cell line often used as a model for human hepatocytes [31], displayed enhancer chromatin upstream and downstream of ANGPTL3 (Figure 2B). It was the only examined cell line that expressed this gene (Figure 2E). However, the steady-state levels of ANGPTL3 RNA in HepG2 cells are $<1 \%$ those of liver. Consistent with this difference in expression, HepG2 had enhancer chromatin near the TSS instead of promoter chromatin and had less enhancer chromatin in its vicinity than did liver. Transcription factor (TF) chromatin immunoprecipitation/DNA sequencing (ChIP-seq) profiles of HepG2 indicated that liver/HepG2 enhancer chromatin regions downstream of ANGPTL3 were bound by broadly expressed and enhancer-related TFs, EP300 and CEBPB; the liver-associated TF HNF4A; and RXRA, which codes for an activator of the liverassociated LXRA/NR1H2 TF (Figure 2C). In addition, HNF4A, RXRA, EP300 and CEBPB were bound within $4 \mathrm{~kb}$ of the DOCK7 TSS in promoter or enhancer chromatin (Figure $2 \mathrm{C}$ ) and also $44 \mathrm{~kb}$ upstream of the DOCK7 TSS in enhancer chromatin seen preferentially in liver (data not shown).

In a study of dyslipidemia patients, a blood lipid-linked Genome-Wide Association Study (GWAS) identified a single nucleotide polymorphism (SNP, rs4240705) that was associated with RXRA and exhibited a significant gene-gene genetic interaction with only one other gene, ANGPTL3 [39]. Interestingly, when we looked at the chromatin state of that SNP, which we found to have no high linkage disequilibrium (LD) proxies, we found that this SNP was embedded in intron 5 of $R X R A$ in enhancer chromatin seen in liver (data not shown). Additionally, we noticed that the ANGPTL3 / DOCK7-related lead SNP (rs12130333) in that study was associated with eight LD proxies $\left(\mathrm{R}^{2}>0.8\right)$, two of which (rs12401837 and rs 12403212) overlap an enhancer chromatin segment that is 44-kb upstream of the DOCK7 TSS and is found preferentially in liver and HepG2 (data not shown). Therefore, yet another liver-associated enhancer chromatin segment might help regulate ANGPTL3, this one in an intergenic region $127 \mathrm{~kb}$ downstream of ANGPTL3 and much closer to the DOCK7 TSS than to the ANGPTL3 TSS. The significant underexpression of $D O C K 7$ in liver (Table 1) and the lack of liver specificity for $A T G 4 C$, the nearest upstream gene to DOCK7 (95 kb upstream; data not shown), make it likely that this is another ANGPTL3-distal enhancer that regulates $A N G P T L 3$.

\section{Liver-specific DNA hypomethylation in a 15-kb liver super-enhancer overlapping ANGPTL8}

The largest amount of both enhancer and promoter chromatin overlapping or adjacent to ANGPTL8 was seen in liver (Figure 3B), which is consistent with liver being the predominant tissue expressing this gene (Figure 1B). Although a database for super-enhancers (dbSuper [40]), does not list liver as having a super-enhancer in this region, this 14.6-kb enhancer/promoter region should qualify as one according to the definition of super-enhancers as $>3 \mathrm{~kb}$ regions consisting mostly of strong enhancer chromatin [41,42]. Much of this ANGPTL8 super-enhancer exhibited liver-specific DNA hypomethylation, which overlapped ANGPTL8's host DOCK6 gene. Adipose, the second highest-expressing tissue also displayed a super-enhancer in this region although adipose tissue expresses this gene at much lower levels than liver (Figure 1B). However, adipose lacked the promoter and enhancer DNA hypomethylation of liver and had enhancer chromatin rather than promoter chromatin near the TSS (Figure 3B \& $\mathrm{D})$, which suggests the importance of these epigenetic features for regulating the levels of expression of ANGPTL8.

Aorta and heart, which have very low or negligible expression of ANGPTL8, exhibited DNA hypomethylation overlapping part of the far-downstream region of the liver super-enhancer. This subregion exhibited high DNA methylation in liver (Figure 3D, pink highlighting), which we propose might favor ANGPTL8 expression. Enhancers and super-enhancers are generally associated with DNA hypomethylation and increased DNA methylation at enhancers is implicated in negatively regulating their activity [43,44], Nonetheless, high DNA methylation of subregions within enhancers has also been correlated with enhancer activity [45,46]. 
Like liver, HepG2 cells had promoter chromatin immediately upstream of the ANGPTL8 TSS as well as much enhancer chromatin in and around ANGPTL8. These chromatin marks correlate with high levels of expression of this gene in HepG2 cultures (Figure 3B \& F). According to ChIP-seq profiles, the liver-associated TF HNF4A and RXRA bound to promoter or enhancer chromatin near or adjacent to ANGPTL8 in HepG2 (Figure 3C). The ChIP-seq evidence for in vivo binding of RXRA to a region $\sim 2.8 \mathrm{~kb}$ upstream of the ANGPTL8 TSS in HepG2 cells matches the in vitro evidence for this binding [47]. RXRA and HNF4A were bound to another HepG2 enhancer chromatin region $\sim 1 \mathrm{~kb}$ further upstream (blue highlighting, Figure $3 \mathrm{C}$ ). Additional HepG2 ChIPseq profiles showed that RNA polymerase II (which often catalyzes low-level transcription from enhancers [48]) and enhancer-associated CEBPB and EP300 were bound nearby while the chromatin looping associated protein CCCTC-binding factor (CTCF), which is involved in enhancer-promoter looping, and MAFK, a broadly expressed enhancer-associated TF, were bound to the ANGPTL8-downstream putative enhancer region. Although bisulfiteseq data for HepG2 cells are not available for evaluating DNA methylation around this gene, RRBS profiles (which provide much lower-coverage than bisulfite-seq) are publicly available for these cells [31]. RRBS revealed DNA hypomethylation in the proximal portion of this enhancer chromatin specifically in HepG2 as well as in hepatocytes and liver (dotted box, Figure 3E).

In HepG2 cells, a predicted insulator is $\sim 7 \mathrm{~kb}$ downstream of the DOCK6 TSS and $\sim 11 \mathrm{~kb}$ downstream of the HepG2 enhancer region according to ENCODE chromatin segmentation tracks (data not shown, [29,49]). Similar DOCK6 RNA levels were found in HepG2, lung fibroblasts (NHLF), epidermal keratinocytes (NHEK), embryonic stem cells (H1 ESC), myoblasts, LCL and erythroleukemia (K562) cell cultures (data not shown [29]) even though only HepG2 cells had ANGPTL8-linked enhancer chromatin. The predicted insulator could minimize the interaction of the ANGPTL8-associated enhancer chromatin with the DOCK6 promoter thereby preventing overexpression of DOCK6 (Table 1).

\section{Tissue-specific enhancer chromatin \& DNA hypomethylation associated with ANGPTL1, ANGPTL2, ANGPTL4 \& ANGPT1}

Four other ANGPT/ANGPTL genes, ANGPTL1, ANGPTL2, ANGPTL4 and ANGPT1, exhibited enhancer and promoter chromatin preferentially in tissues and cell cultures in which these genes are most highly expressed (Supplementary Figures S1-S4). Complicating the analysis of enhancer chromatin is that ANGPTL1 and ANGPTL2 (like ANGPTL3 and ANGPTL8) are expressed as transcripts antisense to their host genes, RALGPS2 and RALGPS1, respectively. Differences in tissue-specific expression (Supplementary Table 1) of these two intronic-gene/host-gene pairs allow the probable assignment of many of the enhancer chromatin segments to one or the other genes of these gene pairs. For example, the presence of enhancer chromatin far upstream of ANGPTL1 and downstream of its host gene correlated much better with expression of ANGPTL1 than with that of the host gene (Supplementary Figure 1). However, there was not always a strict correlation between the amount of enhancer chromatin and the level of gene expression. For example, some of the liver/HepG2-specific enhancer chromatin associated with ANGPTL4 (Supplementary Figure S3B, [yellow dotted boxes]) was not observed in adipose tissue and normal human epidermal keratinocyte (NHEK) cell cultures despite the higher expression of this gene in adipose and NHEK than in liver and HepG2 cells (Figure $1 \&$ Supplementary Figure S3D \& E).

ANGPT1/ANGPTL1, 2 and 4 expression also correlated with tissue-specific DNA hypomethylation in enhancer or promoter chromatin (Supplementary Figures S1-4). In the middle of the long intron 1 of ANGPT1 were two short hypomethylated DMRs (0.6 and $2 \mathrm{~kb}$ ) that overlap short enhancer or intragenic promoter chromatin regions in aorta and ovary; both tissues moderately express this gene (arrows, Supplementary Figure S4B and C). In contrast, ANGPTL1, ANGPTL2 and ANGPTL4 displayed hypomethylated DMRs that cover just a small portion of their tissue-specific enhancer chromatin (Supplementary Figures S1-3). The DMRs' enhancer subregion specificity could not be explained just by different intensities of H3K27ac or H3K4me1 signals (data not shown).

\section{Atherosclerosis-associated changes in DNA methylation at ANGPT2 \& the host gene for miR-145 that probably affect $A N G P T 2$ RNA levels}

$A N G P T 2$ resides in antisense orientation within intron 12 of the long isoform (237 kb) of $M C P H 1$, a broadly expressed, DNA damage-response gene (Figure 4A). Aorta has higher levels of RNA from this gene than most other tissues although the levels are still only moderate (Figures $1 \mathrm{~A} \& 4 \mathrm{~F}$ ). ANGPT2 regulates normal angiogenesis. Its encoded protein also affects the progression of various diseases but through complex mechanisms that involve regulation of vascular homeostasis and inflammation as well as angiogenesis [9,50]. Tissue-specific differences in 
(A)

(B)

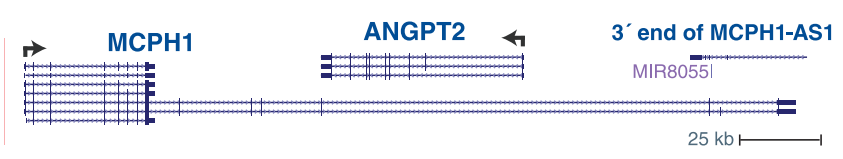

Chromatin state segmentation

$\square \square$ Str, Wk, Biv Prom $\square \square \square \square$ Str, Wk, Biv Enh or Enh/Prom $\square \square$ Txn-chrom $\quad$ Low signal (white) $\square \square \square$ Repr
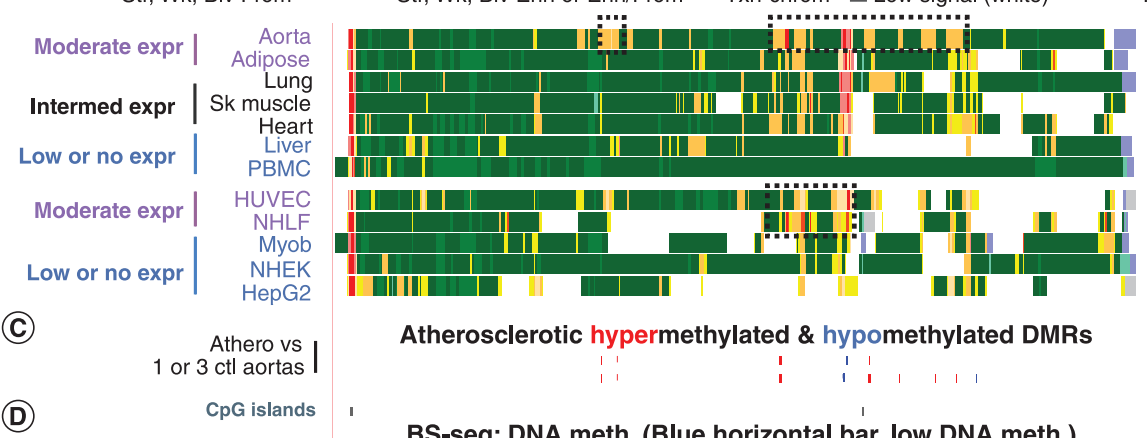

Atherosclerotic hypermethylated \& hypomethylated DMRs

BS-seq: DNA meth. (Blue horizontal bar, low DNA meth.)

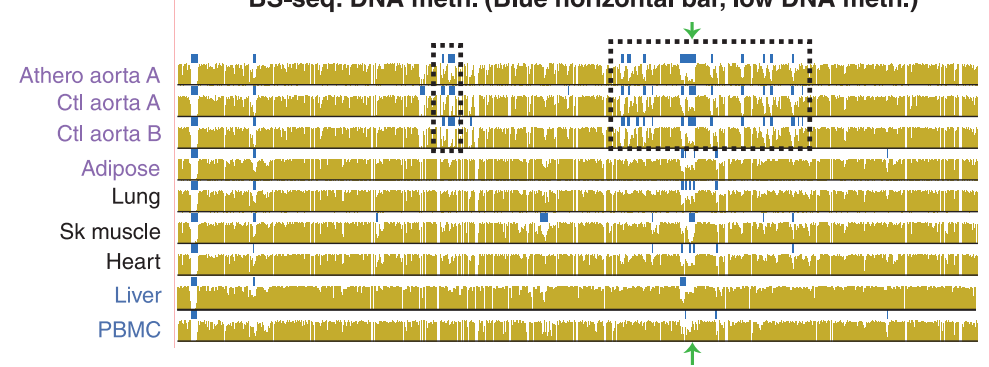

(E)

RNA-seq (not strand-specific for tissues; strand-specific for cultures)

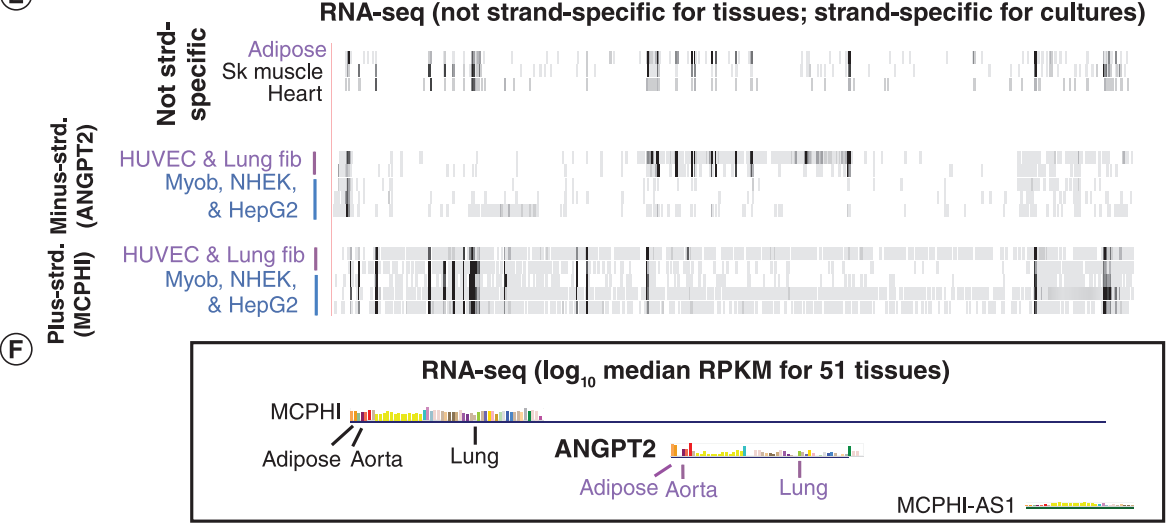

(G)

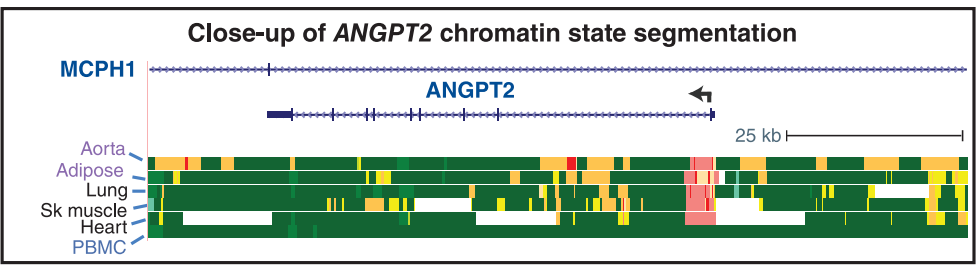

Figure 4. ANGPT2 displays atherosclerosis-associated changes in DNA methylation in aorta. (A) ANGPT2 resides in an intron of long isoforms of Microcephalin 1, MCPH1 (chr8:6,258,632-6,509,865). (B) Chromatin state segmentation as in Figure 2. (C) Statistically significant DMRs (hypermethylated in red or hypomethylated in blue) in an atherosclerotic aorta sample versus control aorta from the same individual or vs three control aorta samples as determined from analysis of bisulfite-seq profiles. (D) CpG islands and bisulfite-seq profiles; green arrow, exon-1 subregion previously found to be hypomethylated in cancers [51]. (E) and (F), RNA-seq profiles as described for Figure 2. (G) Zoomed-in view of chromatin segmentation tracks from panel (B) showing just ANGPT2 and most of the aorta-specific enhancer regions around it (chr8 : 6, 340, 035-6, 456, 867). Dotted boxes, regions of aorta-specific DNA hypomethylation and enhancer chromatin; green arrows, previously studied exon 1 region described in the text. DMR: Differentially methylated region; fib: Fibroblast; HepG2: Hepatocarcinoma cell; HUVEC: Human umbilical vein endothelial cell; LCL: Lymphoblastoid cell line; Myob: Myoblast; PBMC: Peripheral blood mononuclear cells; RRBS: Reduced representation bisulfite sequencing; Sk muscle: Skeletal muscle; RPKM: Reads per kilobase million. 
promoter and enhancer chromatin profiles around ANGPT2 generally correlated with the gene's tissue-dependent differences in steady-state levels of its mRNA (Figure 4B, E-G). The major exception was that aorta had more enhancer chromatin and hypomethylated DMRs in this region than did adipose, which, surprisingly, had higher steady-state levels of ANGPT2 RNA (Figure 4F; Supplementary Table 1). This is likely due to differential mRNA stability (see description of miR-145 below).

Using published DNA methylation profiles [20,28], we determined statistically significant atherosclerosisassociated DMRs in aorta (9220 total DMRs [35]). Remarkably, ANGPT2 had nine atherosclerosis-related DMRs in its vicinity. Seven of these were hypermethylated DMRs and overlapped ANGPT2 aorta-specific enhancer chromatin within the body of the long isoform of $M C P H 1$ (Figure 4C, red bars). In addition, we found an atherosclerosis-associated hypomethylated DMR immediately downstream of the ANGPT2 TSS in a region of weak promoter chromatin in aorta (Figure 4C, blue bars). The hypomethylation was $\sim 1 \mathrm{~kb}$ downstream of a 65-bp exon-1 subregion (green arrow, Figure 4D) that had been studied by Martinelli et al. [51] and found to be hypomethylated in the subset of chronic lymphocytic leukemias that had features of poorer prognosis and exhibited higher expression of ANGPT2.

MicroRNAs play critical roles in atherosclerosis [52]. A microRNA, miR-145, is expressed at extraordinarily high levels in aorta and other smooth muscle-rich tissues (Supplementary Table 1) and targets ANGPT2, among other genes, in breast cancer cells [53,54] and in brain microvascular endothelial cells [55]. We found many statistically significant atherosclerosis-associated hypermethylated DMRs near miR-145-encoding sequences in aorta (Figure 5C); however, the coordinates of the gene encoding this miRNA are uncertain. Most of these DMRs are embedded in CARMN, the long non-coding RNA (lncRNA) gene that overlaps DNA sequences coding for miR-145 (hsamiR-145-5p) and multiple lncRNA isoforms (Ensembl isoforms in Figure 5A) necessary for differentiation of cardiac precursor cells [56]. These atherosclerosis-linked hypermethylated DMRs are located in enhancer chromatin regions that were hypomethylated in normal aorta relative to other normal tissues (Figure 5C \& D). There was no evidence for atherosclerosis-linked DNA hypermethylation in the putative MIR145 promoter region (green arrow, Figure 5G) described in many studies of cancer-associated 'promoter' methylation of MIR145 often coupled with lower levels of miR-145 [53,57,58]. This putative promoter region extends from $-1.4 \mathrm{~kb}$ to the beginning of the miR-145-encoding sequences and was shown to drive expression of a reporter gene in transfection experiments [59].

Cancer-associated DNA hypermethylation in this subregion might actually be acting on enhancer chromatin (both overlapping CARMN and a distinct region starting $\sim 10 \mathrm{~kb}$ downstream of the gene) rather than on the promoter(s) for MIR145. The main promoter region of the $26-\mathrm{kb} C A R M N$ is $22-23 \mathrm{~kb}$ upstream of the 88 bp RefSeq MIR145 and the $2.5 \mathrm{~kb}$ Ensembl MIR145 (ENSEMBL00000602315). In addition, none of the ten $C A R M N$-overlapping Ensembl transcript isoforms that were found to be expressed in adult cardiac precursor cells [60] overlap sequences encoding miR-143, which, like miR-145, is very highly expressed in aorta (Figure 5A \& $\mathrm{H})$. Nonetheless, several of these Ensembl isoforms have been mapped to an $\sim 17.5-\mathrm{kb}$ Ensembl gene that is named MIR143HG (Figure 5H). As shown in Figure 5, strong promoter chromatin for the examined miR-145 and/or miR-143 expressing tissues and cell cultures is seen only at the $5^{\prime}$ end of CARMN, with the exception of heart, which also displays promoter chromatin $4.3 \mathrm{~kb}$ upstream of miR-145-encoding sequences. Examination of RNAseq intensities across CARMN and evaluation of $5^{\prime}$ cap analysis of gene expression (CAGE) profiles of cell cultures (Figure 5F \& G and data not shown) suggest a TSS only at the $5^{\prime}$ end of CARMN for osteoblasts, myoblasts and an LCL. However, lung fibroblasts may use a TSS for MIR145 or MIR143 closer to the sequences encoding these miRNAs or have different stability of precursors to these miRNAs (Figure 5G). We found that the tissue expression profiles for the Ensembl 106-bp MIR143, $2.5 \mathrm{~kb}$ MIR145 and the composite $17.5 \mathrm{~kb}$ Ensembl MIR143HG are very similar (Figure $5 \mathrm{H}$ ). Our analysis suggests that the aorta/ovary/heart super-enhancer spanning the 26-kb CARMN region upregulates MIR143 and MIR 45 whatever the exact locations of the probably alternate TSS for these miRNA genes, and is likely to be susceptible to disease-related downregulation by DNA hypermethylation.

\section{Discussion}

In this study we describe positive correlations between tissue-specific expression, enhancer chromatin and DNA hypomethylation for the atherosclerosis-related genes ANGPTL3, ANGPTL8, ANGPT2, ANGPTL1 and ANGPTL2, which are embedded within introns of larger genes, and for the stand-alone ANGPT1 and ANGPTL4 genes (Figure 6). The intricate tissue-specific epigenetic profiles for these genes and their surrounding sequences can reflect their multiple regulatory roles in normal tissues. For example, we found that the liver-specific expression of $A N G P T L 3$ and $A N G P T L 8$ is accompanied by liver-specific DNA hypomethylation overlapping some (ANGPTL3) 
(A)
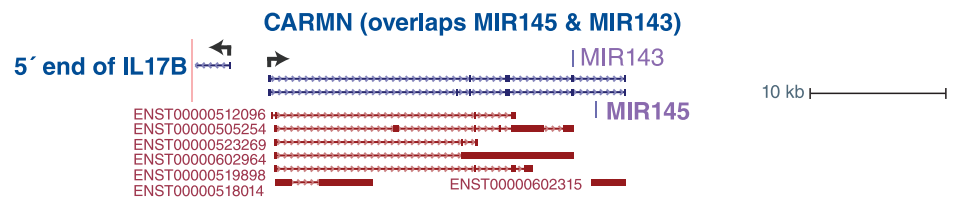

(B)

Chromatin state segmentation

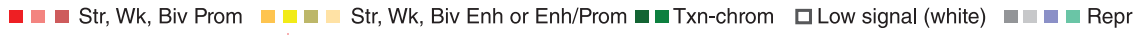



(C)

(D)

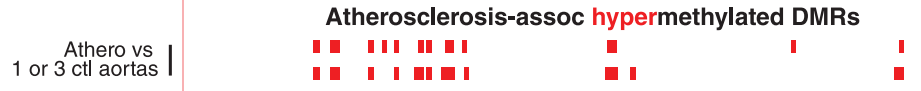

BS-seq: DNA meth. (Blue horizontal bar, low DNA meth.)

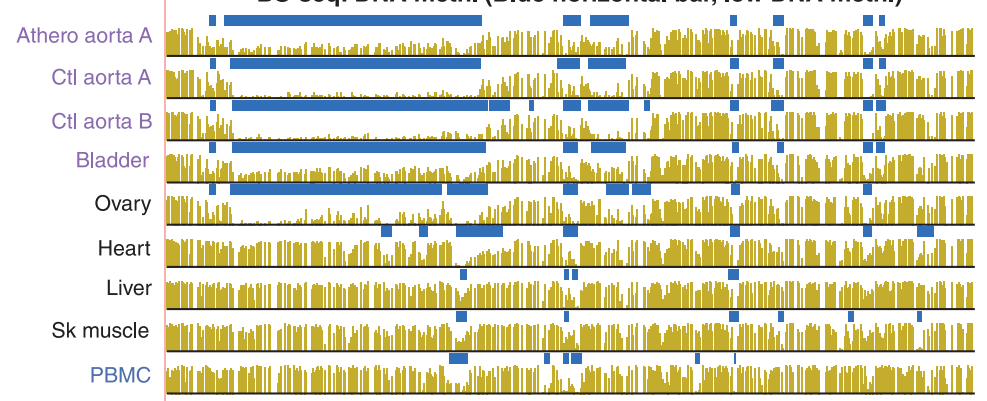

(E)

Small RNA-seq of cell cultures (Plus strand)

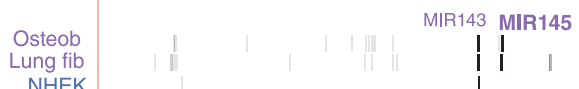

(F)

Long RNA-seq of cell cultures (Plus strand)

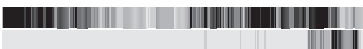

Osteob
Lung fib

NHEK

Breast

Long RNA-seq of tissues (not strand-specific)

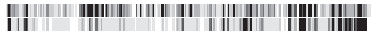

CAGE (5' cap mapping; plus-strand)

(G)

$$
\checkmark
$$

(H)

Osteob
Lung fib
NHEK

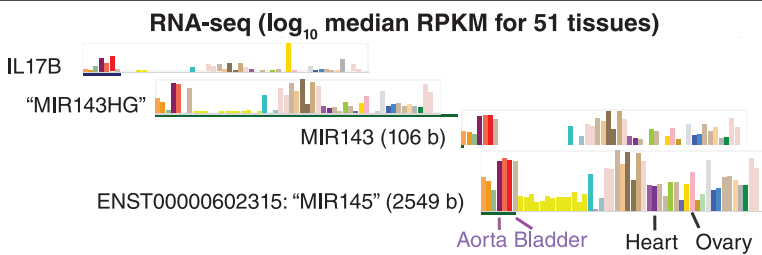

Figure 5. The gene encoding miR-145 is embedded in an aorta/ovary/heart/osteoblast/lung fibroblast super-enhancer that overlays atherosclerosis-associated hypermethylated regions. (A) Two RefSeq isoforms (CARMN) that overlap the miR-145- and miR-143-encoding sequences and six of the ten Ensembl isoforms in this region, including the only Ensembl isoform that overlaps miR-143-encoding DNA (chr5 : 148, 781, 015-148, 839, 604). (B) Chromatin state segmentation as in Figure 2. (C) Atherosclerosis-associated DMRs as in Figure 4. (D) Bisulfite-seq as in Figure 2. (E) Short RNA-seq $(<0.20 \mathrm{~kb})$; the position of the sequences encoding the pre-miRNAs is indicated. (F), RNA-seq ('Long' RNA-seq for RNAs $>0.2 \mathrm{~kb}$ ) as in Figure 2 except that the vertical viewing range was 0 -300 instead of 0-30 as in other figures. (G) CAGE (TSS from Hidden Markov Modeling for pooled replicates; [29]); green arrow, the position of the putative 1.4-kb MIR145 promoter region mentioned in the text. $(\mathrm{H})$ Median RPKM values from RNA-seq for tissues. The CpG islands are not shown because there were none in this region that fit the definition used by the UCSC Genome Browser [30].

CAGE: 5' cap analysis of gene expression; DMR: Differentially methylated region; HUVEC: Human umbilical vein endothelial cell; PBMC: Peripheral blood mononuclear cells; Sk muscle: Skeletal muscle; RPKM: Reads per kilobase million. 


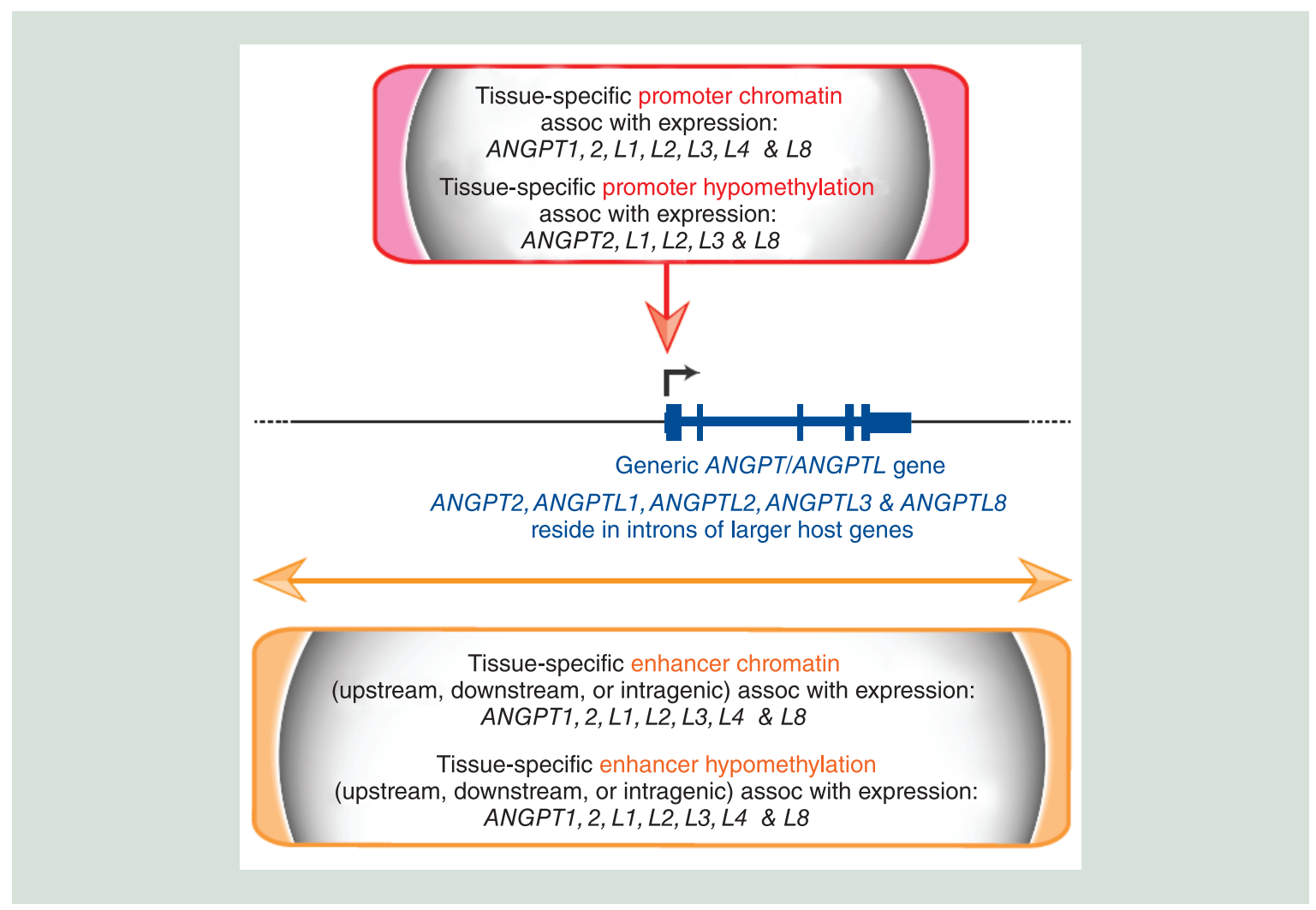

Figure 6. A cartoon summarizing correlations of tissue-specific DNA hypomethylation, active promoter or enhancer chromatin with tissue-specific expression of the studied ANGPT or ANGPTL genes. 2, L1, L2, L3, L4 and L8 denote ANGPT2, ANGPTL1, ANGPTL2, ANGPTL3, ANGPTL4 and ANGPTL8.

Assoc: Associated.

or much (ANGPTL8) liver-specific enhancer chromatin inside their host genes. The nonuniform distribution of DNA hypomethylation along their enhancer chromatin segments suggests functionally important subregions of these enhancers [44,45,61]. Moreover, our comparisons of the tissue-and cell type-specificity of the epigenetics and transcriptomics in gene neighborhoods helps assign enhancers to their target genes in vivo in cases of geneswithin-genes like the ANGPTL3 /DOCK7 gene pair. The liver- and HepG2-specific enhancer chromatin seen downstream of $A N G P T L 3$ and within the body of the DOCK7 gene is probably upregulating only ANGPTL3, given the liver-preferential expression of this gene and the underexpression of DOCK 7 in liver. Similar findings for the ANGPTL8/DOCK6 gene pair suggest that increases in host gene expression for such inversely expressed intronic-gene/host-gene pairs might be a mechanism to down-modulate expression of their intronic genes.

In contrast, some of the enhancer chromatin near or at $A N G P T 2$ and its oppositely oriented host gene $M C P H 1$ might represent enhancers shared between these two genes because we found a significant positive correlation between the presence of this enhancer chromatin and expression of both of these genes among different tissues. Our finding that there is a negative correlation between ANGPTL3 and DOCK7 expression is consistent with results from a previous study of pluripotent cells [62]. Unlike the directly correlated ANGPT2 and MCPH1 expression that we described in Results, Kopparapu et al. [24] reported an inverse relationship between expression of these two overlapping genes in chronic lymphocytic leukemia blood samples. Such an inverse relationship might exist only in cancers, given the extensive epigenetic derangements that accompany oncogenesis and tumor progression. Moreover, we observed that blood had the lowest expression of both ANGPT2 and host gene MCPH1 relative to other tissues (Supplementary Table 1) and exhibited tissue-specific hypermethylation at the promoter region of ANGPT2 (Figure 4). These findings are also inconsistent (at least in normal tissues) with the hypothesis of Kopparapu et al. [24] that MCPH1 protein is involved in recruiting DNA methyltransferases for establishing such hypermethylation. 
Comparisons of the DNA or chromatin epigenetics of many different normal tissues and cell types, like those made in the present study, provide insights into transcription regulatory elements that can be difficult to obtain from analysis of expression quantitative trait loci (eQTLs) or GWAS SNPs. For example, ANGPTL3 has hundreds of SNPs throughout the DOCK7 host gene that are associated with coronary artery disease and in very strong LD [63]. In a study of adipose eQTLs and GWAS SNPs for high-density lipoprotein cholesterol that was focused on the neighborhood of ANGPTL8 [47], only a minority of the $\sim 20$ index SNPs or their LD proxies overlapped enhancer chromatin in liver, and these were all immediately upstream of the ANGPTL8 promoter. Our analysis of epigenetic profiles indicates that the enhancer chromatin segments located within the ANGPTL8 gene body and far downstream of the gene also need to be considered for their possible contribution to upregulation of $A N G P T L 8$ expression in liver. Moreover, the $\sim 15-\mathrm{kb}$ enhancer chromatin region that spans ANGPTL8 intragenic, upstream and downstream sequences and that overlaps three liver-specific subregions of DNA hypomethylation (Figure 3) probably functions as a super-enhancer [41,42]. Enhancer chromatin segments within the super-enhancer may be involved in interactions potentiating their especially strong enhancer activity and making them highly responsive to various types of cell signaling [64].

Much of the relevance of ANGPTL8 protein to atherosclerosis involves its interaction with full-length ANGPTL3 or the biologically active C-terminal ANGPTL3 fragment, which it helps to generate $[6,14,18]$. ANGPTL3, which is specifically expressed in liver, plays critical roles in determining the levels of cholesterol and triglycerides in circulating blood [15]. It has also been reported to induce endothelial cell adhesion and migration, which are correlated with angiogenesis [65]. The association of a cardiovascular phenotype (significant reduction in plasma lipids and coronary artery disease) with heterozygous loss-of-function from ANGPTL3 mutations [16] suggests that normal expression of this gene is especially closely regulated. Both ANGPTL8 and ANGPTL3 enhancer chromatin displayed binding of RXRA and the liver-associated TF HNF4A in HepG2 cells. RXRA heterodimerizes with the liver-associated TF LXRA to give a ligand-dependent TF that is sensitive to lipid levels [66]. Despite these TFs also binding near the DOCK7 TSS, DOCK7 is underexpressed in liver relative to other tissues (Table 1). Therefore, these liver-associated transcription regulatory elements far from ANGPTL3 and closer to the $5^{\prime}$ end of DOCK7 probably upregulate transcription from $A N G P T L 3$ and not from DOCK7. Moreover, the evidence for binding of a RXRA:LXRA heterodimer to liver-specific enhancer chromatin in the neighborhood of ANGPTL3/DOCK7 suggests that the previously demonstrated induction of $A N G P T L 3$ by LXR-selective agonists likely involves binding to the ANGPTL3 enhancer as well as to the promoter [15,66].

Using publicly available DNA methylation profiles [20] and our own more stringently determined atherosclerosisrelated DMRs [35], we found that ANGPT2 was the only ANGPT/ANGPTL gene that contains multiple and statistically significant atherosclerosis-associated DMRs. ANGPT2 protein, which is implicated in various diseases including atherosclerosis, has been related to endothelial cell-directed vascular remodeling and angiogenesis as well as to increasing plasma triglyceride levels and formation of fatty streaks in aorta [7]. However, unlike ANGPT1, ANGPT2 can destabilize vascular endothelial monolayers as well as stabilize them depending on whether or not the surrounding tissue is inflamed [7]. Studies of cancer and brain microvascular endothelial cells indicate that ANGPT2 expression can be regulated at the post-transcriptional level by miR-145 [26,55]. Furthermore, miR-145 is important in atherosclerosis because of its role in controlling genes involved in SMC phenotype-switching [67,68]. The approximately eightfold higher levels of miR-145 in aorta than in adipose tissue could explain the twofold lower steady-state levels of ANGPT2 RNA in aorta despite the presence of much more enhancer chromatin at or near $A N G P T 2$ in aorta than in adipose tissue.

The large IncRNA gene CARMN, which overlaps the sequences encoding miR-145 and miR-143 and a smooth muscle/cardiac muscle super-enhancer, had many atherosclerosis-associated hypermethylated DMRs (Figure 5). This atherosclerosis-linked hypermethylation at the super-enhancer is predicted to decrease enhancer activity because extensive DNA hypermethylation at enhancers is generally associated with lower expression of their associated genes [43-45]. Less miR-145/143 in SMCs [69], the main cell type in aorta, could mean less of these miRNAs in the neighboring endothelial aorta cells because SMCs can transfer miR-145/143 to endothelial cells to modulate their phenotype [70]. Therefore, atherosclerosis-linked CARMN hypermethylation and ANGPT2 intron 1 hypomethylation (Figure 4) [71] could act at the translational and transcriptional levels respectively to increase ANGPT2 protein levels. In contrast, the hypermethylation of ANGPT2 at multiple atherosclerosis-associated DMRs overlapping aorta enhancer chromatin is predicted to decrease expression of this gene in atherosclerotic aorta. These seemingly opposite results might reflect the varied functions of $A N G P T 2$ at different stages of atherosclerosis [10] and the cellular heterogeneity in the samples. Indeed, a caveat in many of our epigenetic analyses 
is that tissue heterogeneity can lead to underestimation of specific epigenetic differences within the tissues, which seems to be the case for tissue-specific DNA methylation changes in several of the studied genes at enhancer or promoter chromatin in adipose tissue (Figure $4 \&$ Supplementary Figures 1, $2 \& 4$ ).

The central role of enhancers in the regulation of tissue-specific and disease-related transcription [72] is illustrated for $A N G P T / A N G P T L$ genes by a study of zebrafish angptl4 [73]. This gene's mammalian counterpart is implicated in the regulation of lipid and glucose metabolism, energy homeostasis, redox regulation, angiogenesis, inflammation, endothelial cell integrity, cancer and obesity [6,74]. Intestine-specific expression of a reporter transgene in zebrafish is driven by the intronic angptl4 enhancer but not by this gene's promoter [73]. This intestine-specific enhancer appears to be the most important cis-regulatory angptl4 element for microbiota-associated peripheral fat-storage in zebrafish. In HepG2 cells, enhancer chromatin in the last exon of ANGPTL4 was demonstrated to form a stable higher order chromatin structure with the promoter of the gene in response to glucocorticoid treatment [75], which suggests, along with the current study (Supplementary Figure S4), that gene-body enhancer chromatin is important for expression of this gene in humans too.

Nonetheless, most studies of cancer-linked changes in gene expression and epigenetics at $A N G P T / A N G P T$ and the ANGPT2 mRNA-targeting MIR145 examine just sequences in their known or presumptive promoter region or sequences immediately downstream of the $5^{\prime}$ end of the gene [51,57]. Examining only promoter region methylation at cancer-related genes such as ANGPTL4 and ANGPTL2 in tumors [25,76] overlooks possible oncogenic DNA hypermethylation at enhancer sequences. Such a limited approach to cancer epigenetics at ANGPT/ANGPTL genes is commonly used although it also misses detection of possible oncogenic enhancer DNA hypomethylation that might upregulate neoangiogenesis in stromal tissues near solid cancers. Lack of attention to enhancers also presents a problem in disease-associated DNA methylation studies focusing on reactivation of a hypermethylated promoter by treatment with a global DNA methylation inhibitor. Among the many genes examined in that type of study are MIR145 and ANGPTL2 [51,53,58]. Global demethylating agents can affect the gene of interest not only by trans effects due to methylation changes at other genes but also by cis effects at unexamined enhancers as well as at promoters. An additional complication for MIR145 is the uncertainty as to whether the sequences tested for cancer-linked DNA methylation changes were acting as part of promoter or enhancer elements for the gene. Our findings from evaluating the relationships between tissue-specific gene expression and epigenetics can help to more systematically guide the development of diagnostic and prognostic tools and of DNA or RNA-based therapies for cancers, cardiovascular diseases, and other diseases that are critically affected by ANGPT/ANGPTL-encoded proteins or the $A N G P T 2$-regulatory miR-145 [6,7,77-79].

\section{Conclusion}

The many roles of ANGPT/ANGPTL proteins in development are reflected in their tissue-specific expression. Here, we have shown that these expression profiles can be correlated with tissue-specific epigenetics. Frequent DNA hypomethylation associated with $A N G P T$ or $A N G P T L$ family genes was seen specifically in subregions of tissue-specific enhancer chromatin as well as at promoter chromatin. We have identified likely intragenic and intergenic proximal or distal enhancers that could upregulate transcription of ANGPT / ANGPTL genes, including distal enhancers residing in host genes harboring five of the ANGPT / ANGPTL genes. These enhancer chromatin regions and their DNA methylation status should be considered in future studies of the many relationships of ANGPT / ANGPTL genes to vascular, neoplastic, immunological and metabolic diseases.

\section{Future perspective}

Our research indicates the importance of examining changes in enhancers and DNA methylation associated with $A N G P T$ and $A N G P T L$ genes in future studies of atherosclerosis. In addition, our findings demonstrate the need for future experiments on disease-relevant cell culture and mouse models to test directly for postulated enhancer/promoter interactions, especially for the genes-within-genes that we analyzed, and for proposed microRNA-mRNA interactions. Moreover, it is compelling to extend studies of atherosclerosis-associated changes in DNA methylation in aorta to multiple samples of disease and control aortic arch tissue from different individuals and to include single-cell analysis of both chromatin and DNA epigenetics in aortic SMCs and endothelial cells. Optimally, such epigenetic changes should be documented with single-nucleotide resolution. Given the critical roles ascribed to many of the studied $A N G P T / A N G P T L$ genes in cancer, the proposed future studies of their chromatin and DNA epigenetics compared with their expression in disease models should include examining cancer formation and metastasis as well as atherosclerosis. 


\section{Summary points}

- ANGPT1 and 2 and ANGPTL1, 2, 3, 4 and 8 regulate vascular permeability and remodeling, inflammatory responses and lipid and glucose metabolism. All display tissue-specific enhancer and promoter chromatin that correlates with their expression.

- Five of the small genes encoding these proteins are located in an intron of a large gene and display distal tissue-specific enhancer chromatin that is likely to upregulate the ANGPT/ANGPTL gene rather than the host gene.

- Two of the genes-within-genes (ANGPTL3 /DOCK7 and ANGPTL8 /DOCK6) showed a negative association of expression of the intronic gene and that of the host gene; the other three displayed either a positive association (ANGPT2 /MCPH1) or no significant association (ANGPTL1 /RALGPS2 and ANGPTL2 /RALGPS1).

- Enhancer chromatin that probably regulates ANGPT/ANGPTL genes is newly described for the following gene regions: gene-downstream for ANGPTL3 (at the 1.8-kb AL138847 gene), ANGPTL8, ANGPT2 and ANGPTL2 ; gene-upstream for ANGPTL3, ANGPT2, ANGPTL1, ANGPTL4 and ANGPT1; and intragenic for all seven studied ANGPT/ANGPTL genes.

- Tissue-specific DNA hypomethylation was seen in subregions of the enhancer chromatin for all the genes and also at the promoter region for many of the genes.

- Disease-related changes in the epigenetics of ANGPT/ANGPTL genes were evidenced by the atherosclerosis-associated changes in DNA methylation in enhancer and promoter region sequences at ANGPT2.

- Atherosclerosis-linked changes in DNA methylation were also seen near the DNA sequences encoding miR-145, a miRNA that was previously implicated in regulating ANGPT2 mRNA translation in cancer.

- The loss of normal fine-tuning of expression of ANGPT /ANGPTL genes in atherosclerosis, inflammatory and metabolic diseases, and cancer is likely to include often overlooked changes in enhancer activity.

\section{Supplementary data}

To view the supplementary data that accompany this paper please visit the journal website at: https://www.futuremedicine.com/d oi/suppl/10.2217/epi-2018-0150

Financial \& competing interest disclosure

This research was supported in part by a grant from the NIH (National Center for Advancing Translational Sciences, award number UL1TR001417) and the Louisiana Cancer Center to M Ehrlich. The authors have no other relevant affiliations or financial involvement with any organization or entity with a financial interest in or financial conflict with the subject matter or materials discussed in the manuscript apart from those disclosed.

No writing assistance was utilized in the production of this manuscript.

Ethical conduct of research

Publicly available databases were used for the research.

Open access

This work is licensed under the Attribution-NonCommercial-NoDerivatives 4.0 Unported License. To view a copy of this license, visit http://creativecommons.org/licenses/by-nc-nd/4.0/ 


\section{References}

Papers of special note have been highlighted as: $\bullet$ of interest; $\bullet \bullet$ of considerable interest

1. Vanderburgh JA, Reinhart-King CA. The role of age-related intimal remodeling and stiffening in atherosclerosis. Adv. Pharmacol. 81, 365-391 (2018).

2. Chistiakov DA, Orekhov AN, Bobryshev YV. Endothelial barrier and its abnormalities in cardiovascular disease. Front. Physiol. 6, 365 (2015).

3. Allahverdian S, Chaabane C, Boukais K, Francis GA, Bochaton-Piallat ML. Smooth muscle cell fate and plasticity in atherosclerosis. Cardiovasc. Res. 114(4), 540-550 (2018).

4. Lao KH, Zeng L, Xu Q. Endothelial and smooth muscle cell transformation in atherosclerosis. Curr. Opin. Lipidol. 26(5), 449-456 (2015).

5. Geovanini GR, Libby P. Atherosclerosis and inflammation: overview and updates. Clin. Sci. (Lond.) 132(12), 1243-1252 (2018).

6. Carbone C, Piro G, Merz V et al. Angiopoietin-like proteins inangiogenesis, inflammation and cancer. Int. J. Mol. Sci. 19(2), (2018).

- Key recent review of angiopoietin-like proteins.

7. Saharinen P, Eklund L, Alitalo K. Therapeutic targeting of the angiopoietin-TIE pathway. Nat. Rev. Drug Discov. 16(9), 635-661 (2017).

8. Santulli G. Angiopoietin-like proteins: a comprehensive look. Front. Endocrinol. (Lausanne) 5, 4 (2014).

9. Yu H, Moran CS, Trollope AF et al. Angiopoietin-2 attenuates angiotensin II-induced aortic aneurysm and atherosclerosis in apolipoprotein E-deficient mice. Sci. Rep. 6, 35190 (2016).

10. Trollope AF, Golledge J. Angiopoietins, abdominal aortic aneurysm and atherosclerosis. Atherosclerosis 214(2), 237-243 (2011).

11. Kim MJ, Namkung J, Chang JS, Kim SJ, Park KS, Kong ID. Leptin regulates the expression of angiopoietin-like 6. Biochem. Biophys. Res. Commun. 502(3), 397-402 (2018).

12. Abu-Farha M, Cherian P, Al-Khairi I et al. Plasma and adipose tissue level of angiopoietin-like 7 (ANGPTL7) are increased in obesity and reduced after physical exercise. PLoS ONE 12(3), e0173024 (2017).

13. Morinaga J, Zhao J, Endo M et al. Association of circulating ANGPTL 3, 4, and 8 levels with medical status in a population undergoing routine medical checkups: a cross-sectional study. PLoS ONE 13(3), e0193731 (2018).

14. Quagliarini F, Wang Y, Kozlitina J et al. Atypical angiopoietin-like protein that regulates ANGPTL3. Proc. Natl Acad. Sci. USA 109(48), 19751-19756 (2012).

15. Su X, Peng DQ. New insights into ANGPLT3 in controlling lipoprotein metabolism and risk of cardiovascular diseases. Lipids Health Dis. 17(1), 12 (2018).

16. Dewey FE, Gusarova V, Dunbar RL et al. Genetic and pharmacologic inactivation of ANGPTL3 and cardiovascular disease. N. Engl. J. Med. 377(3), 211-221 (2017).

17. Haller JF, Mintah IJ, Shihanian LM et al. ANGPTL8 requires ANGPTL3 to inhibit lipoprotein lipase and plasma triglyceride clearance. J. Lipid Res. 58(6), 1166-1173 (2017).

18. Chi X, Britt EC, Shows HW et al. ANGPTL8 promotes the ability of ANGPTL3 to bind and inhibit lipoprotein lipase. Mol. Metab. 6(10), 1137-1149 (2017).

19. Daly C, Eichten A, Castanaro C et al. Angiopoietin-2 functions as a Tie2 agonist in tumor models, where it limits the effects of VEGF inhibition. Cancer Res. 73(1), 108-118 (2013).

20. Zaina S, Heyn H, Carmona FJ et al. DNA methylation map of human atherosclerosis. Circ. Cardiovasc. Genet. 7(5), 692-700 (2014).

- Foundational paper for establishing involvement of DNA methylation in atherosclerosis

21. Fernandez-Sanles A, Sayols-Baixeras S, Subirana I, Degano IR, Elosua R. Association between DNA methylation and coronary heart disease or other atherosclerotic events: a systematic review. Atherosclerosis 263, 325-333 (2017).

22. Xu S, Pelisek J, Jin ZG. Atherosclerosis is an epigenetic disease. Trends Endocrinol. Metab. 29(11), 739-742 (2018).

-. Review showing the importance of epigenetic studies for understanding the pathogenetic events occurring in atherosclerosis.

23. Liu R, Leslie KL, Martin KA. Epigenetic regulation of smooth muscle cell plasticity. Biochim. Biophys. Acta 1849(4), 448-453 (2015).

24. Kopparapu PK, Miranda C, Fogelstrand L et al. MCPH1 maintains long-term epigenetic silencing of ANGPT2 in chronic lymphocytic leukemia. FEBS J. 282(10), 1939-1952 (2015).

25. Hsieh HY, Jou YC, Tung CL et al. Epigenetic silencing of the dual-role signal mediator, ANGPTL4 in tumor tissues and its overexpression in the urothelial carcinoma microenvironment. Oncogene 37(5), 673-686 (2018).

26. Lu R, Ji Z, Li X et al. miR-145 functions as tumor suppressor and targets two oncogenes, ANGPT2 and NEDD9, in renal cell carcinoma. J. Cancer Res. Clin. Oncol. 140(3), 387-397 (2014).

-. Key paper that provides the support for our assertion that miR-145 likely targets ANGPT2 RNA in atherosclerotic plaque formation.

27. Guo X, Li D, Chen M et al. miRNA-145 inhibits VSMC proliferation by targeting CD40. Sci. Rep. 6, 35302 (2016). 
28. Roadmap Epigenomics Consortium, Kundaje A, Meuleman W et al. Integrative analysis of 111 reference human epigenomes. Nature 518(7539), 317-330 (2015).

29. ENCODE Project Consortium. A user's guide to the encyclopedia of DNA elements (ENCODE). PLoS Biol. 9(4), e1001046 (2011).

30. Rosenbloom KR, Armstrong J, Barber GP et al. The UCSC genome browser database: 2015 update. Nucleic Acids Res. 43(Database issue), D670-D681 (2015).

31. Varley KE, Gertz J, Bowling KM et al. Dynamic DNA methylation across diverse human cell lines and tissues. Genome Res. 23(3), 555-567 (2013).

32. Gtex Consortium, Laboratory, Data Analysis \& Coordinating Center (LDACC) - Analysis Working Group, Statistical Methods groups - Analysis Working Group et al. Genetic effects on gene expression across human tissues. Nature 550(7675), 204-213 (2017).

33. Wang ET, Sandberg R, Luo S et al. Alternative isoform regulation in human tissue transcriptomes. Nature 456(7221), 470-476 (2008).

34. Lacey MR, Baribault C, Ehrlich M. Modeling, simulation and analysis of methylation profiles from reduced representation bisulfite sequencing experiments. Stat. Appl. Genet. Mol. Biol. 12(6), 723-742 (2013).

35. Lacey M, Baribault C, Ehrlich KC, Ehrlich M. Atherosclerosis-associated differentially methylated regions can reflect the disease phenotype and are often at enhancers. Atherosclerosis 280, 183-191 (2019).

-• Evidence for down-modulation of enhancers by DNA hypermethylation in atherosclerotic aorta

36. Song Q, Decato B, Hong EE et al. A reference methylome database and analysis pipeline to facilitate integrative and comparative epigenomics. PLoS ONE 8(12), e81148 (2013).

37. Kersey PJ, Allen JE, Allot A et al. Ensembl Genomes 2018: an integrated omics infrastructure for non-vertebrate species. Nucleic Acids Res. 46(D1), D802-D808 (2018).

38. Deniz E, Erman B. Long noncoding RNA (lincRNA), a new paradigm in gene expression control. Funct. Integr. Genomics 17(2-3), 135-143 (2017).

39. Ma L, Ballantyne CM, Belmont JW, Keinan A, Brautbar A. Interaction between SNPs in the RXRA and near ANGPTL3 gene region inhibits apoB reduction after statin-fenofibric acid therapy in individuals with mixed dyslipidemia. J. Lipid Res. 53(11), 2425-2428 (2012).

40. Khan A, Zhang X. dbSUPER: a database of super-enhancers in mouse and human genome. Nucleic Acids Res. 44(D1), D164-D171 (2016).

41. Whyte WA, Orlando DA, Hnisz D et al. Master transcription factors and mediator establish super-enhancers at key cell identity genes. Cell 153(2), 307-319 (2013).

42. Parker SC, Stitzel ML, Taylor DL et al. Chromatin stretch enhancer states drive cell-specific gene regulation and harbor human disease risk variants. Proc. Natl Acad. Sci. USA 110(44), 17921-17926 (2013).

43. Petell CJ, Alabdi L, He M, San Miguel P, Rose R, Gowher H. An epigenetic switch regulates de novo DNA methylation at a subset of pluripotency gene enhancers during embryonic stem cell differentiation. Nucleic Acids Res. 44(16), 7605-7617 (2016).

44. Heyn H, Vidal E, Ferreira HJ et al. Epigenomic analysis detects aberrant super-enhancer DNA methylation in human cancer. Genome Biol. 17, 11 (2016).

45. Ehrlich KC, Paterson HL, Lacey M, Ehrlich M. DNA hypomethylation in intragenic and intergenic enhancer chromatin of muscle-specific genes usually correlates with their expression. Yale J. Biol. Med. 89(4), 441-455 (2016).

- Bioinformatics analysis indicating the association of tissue-specific intragenic and intergenic enhancers with tissue-specific gene expression.

46. Charlet J, Duymich CE, Lay FD et al. Bivalent regions of cytosine methylation and H3K27 acetylation suggest an active role for DNA methylation at enhancers. Mol. Cell 62(3), 422-431 (2016).

47. Cannon ME, Duan Q, Wu Y et al. Trans-ancestry fine mapping and molecular assays identify regulatory variants at the ANGPTL8 HDL-C GWAS locus. G3 (Bethesda) 7(9), 3217-3227 (2017).

48. Mousavi K, Zare H, Dell'orso $\mathrm{S}$ et al. eRNAs promote transcription by establishing chromatin accessibility at defined genomic loci. Mol. Cell 51(5), 606-617 (2013).

49. Ernst J, Kheradpour P, Mikkelsen TS et al. Mapping and analysis of chromatin state dynamics in nine human cell types. Nature 473(7345), 43-49 (2011).

50. Schlosser K, Taha M, Deng Y, McIntyre LA, Mei SHJ, Stewart DJ. High circulating angiopoietin-2 levels exacerbate pulmonary inflammation but not vascular leak or mortality in endotoxin-induced lung injury in mice. Thorax 73(3), 248-261 (2018).

51. Martinelli S, Kanduri M, Maffei R et al. ANGPT2 promoter methylation is strongly associated with gene expression and prognosis in chronic lymphocytic leukemia. Epigenetics 8(7), 720-729 (2013).

52. Novak J, Olejnickova V, Tkacova N, Santulli G. Mechanistic role of microRNAs in coupling lipid metabolism and atherosclerosis. Adv. Exp. Med. Biol. 887, 79-100 (2015).

53. Liu SY, Li XY, Chen WQ et al. Demethylation of the MIR145 promoter suppresses migration and invasion in breast cancer. Oncotarget 8(37), 61731-61741 (2017). 
54. Kozomara A, Griffiths-Jones S. miRBase: integrating microRNA annotation and deep-sequencing data. Nucleic Acids Res. 39(Database issue), D152-D157 (2011).

55. Ren L, Wei C, Li K, Lu Z. LncRNA MALAT1 up-regulates VEGF-A and ANGPT2 to promote angiogenesis in brain microvascular endothelial cells against oxygen-glucose deprivation via targeting miR-145. Biosci. Rep. doi:10.1042/BSR20180226 (2018) (Epub ahead of print).

56. Ounzain S, Micheletti R, Arnan C et al. CARMEN, a human super enhancer-associated long noncoding RNA controlling cardiac specification, differentiation and homeostasis. J. Mol. Cell. Cardiol. 89(Pt A), 98-112 (2015).

57. Harada K, Baba Y, Ishimoto T et al. Suppressor microRNA-145 is epigenetically regulated by promoter hypermethylation in esophageal squamous cell carcinoma. Anticancer Res. 35(9), 4617-4624 (2015).

58. Suh SO, Chen Y, Zaman MS et al. MicroRNA-145 is regulated by DNA methylation and $\mathrm{p} 53$ gene mutation in prostate cancer. Carcinogenesis 32(5), 772-778 (2011).

59. Sachdeva M, Zhu S, Wu F et al. p53 represses c-Myc through induction of the tumor suppressor miR-145. Proc. Natl Acad. Sci. USA 106(9), 3207-3212 (2009).

60. Plaisance I, Perruchoud S, Fernandez-Tenorio M et al. Cardiomyocyte lineage specification in adult human cardiac precursor cells via modulation of enhancer-associated long noncoding RNA expression. JACC Basic Transl. Sci. 1(6), 472-493 (2016).

61. Chandra S, Terragni J, Zhang G et al. Tissue-specific epigenetics in gene neighborhoods: myogenic transcription factor genes. Hum. Mol. Genet. 24(16), 4660-4673 (2015).

62. Pashos EE, Park Y, Wang X et al. Large, diverse population cohorts of hiPSCs and derived hepatocyte-like cells reveal functional genetic variation at blood lipid-associated loci. Cell Stem Cell 20(4), 558-570 e510 (2017).

63. Oldoni F, Palmen J, Giambartolomei C et al. Post-GWAS methodologies for localisation of functional non-coding variants: ANGPTL3. Atherosclerosis 246, 193-201 (2016).

64. Hnisz D, Schuijers J, Lin CY et al. Convergence of developmental and oncogenic signaling pathways at transcriptional super-enhancers. Mol. Cell 58(2), 362-370 (2015).

65. Camenisch G, Pisabarro MT, Sherman D et al. ANGPTL3 stimulates endothelial cell adhesion and migration via integrin alpha vbeta 3 and induces blood vessel formation in vivo. J. Biol. Chem. 277(19), 17281-17290 (2002).

66. Kaplan R, Zhang T, Hernandez M et al. Regulation of the angiopoietin-like protein 3 gene by LXR. J. Lipid Res. 44(1), 136-143 (2003).

67. Sala F, Aranda JF, Rotllan N et al. MiR-143/145 deficiency attenuates the progression of atherosclerosis in Ldlr-/-mice. Thromb. Haemost. 112(4), 796-802 (2014).

68. Zhang YN, Xie BD, Sun L et al. Phenotypic switching of vascular smooth muscle cells in the 'normal region' of aorta from atherosclerosis patients is regulated by miR-145. J. Cell. Mol. Med. 20(6), 1049-1061 (2016).

69. Cordes KR, Sheehy NT, White MP et al. miR-145 and miR-143 regulate smooth muscle cell fate and plasticity. Nature 460(7256), 705-710 (2009).

-• Key early paper relating mir-143/145 expression to changes in aorta smooth muscle changes seen during atherosclerotic plaque formation.

70. Climent M, Quintavalle M, Miragoli M, Chen J, Condorelli G, Elia L. TGFbeta triggers miR-143/145 transfer from smooth muscle cells to endothelial cells, thereby modulating vessel stabilization. Circ. Res. 116(11), 1753-1764 (2015).

71. Anastasiadou C, Malousi A, Maglaveras N, Kouidou S. Human epigenome data reveal increased CpG methylation in alternatively spliced sites and putative exonic splicing enhancers. DNA Cell Biol. 30(5), 267-275 (2011).

72. Heinz S, Romanoski CE, Benner C, Glass CK. The selection and function of cell type-specific enhancers. Nat. Rev. Mol. Cell Biol. 16(3), 144-154 (2015).

73. Camp JG, Jazwa AL, Trent CM, Rawls JF. Intronic cis-regulatory modules mediate tissue-specific and microbial control of angptl4/fiaf transcription. PLoS Genet. 8(3), e1002585 (2012).

74. Kobyliak N, Virchenko O, Falalyeyeva T. Pathophysiological role of host microbiota in the development of obesity. Nutr. J. 15, 43 (2016).

75. Nakamoto M, Ishihara K, Watanabe T et al. The glucocorticoid receptor regulates the ANGPTL4 gene in a CTCF-mediated chromatin context in human hepatic cells. PLoS ONE 12(1), e0169225 (2017).

76. Kikuchi R, Tsuda H, Kozaki K et al. Frequent inactivation of a putative tumor suppressor, angiopoietin-like protein 2, in ovarian cancer. Cancer Res. 68(13), 5067-5075 (2008).

77. Chadwick AC, Evitt NH, Lv W, Musunuru K. Reduced blood lipid levels with in vivo CRISPR-Cas9 base editing of ANGPTL3. Circulation 137(9), 975-977 (2018).

78. Lin Y, Chen F, Shen L et al. Biomarker microRNAs for prostate cancer metastasis: screened with a network vulnerability analysis model. J. Transl. Med. 16(1), 134 (2018).

79. Zheng W, Zhou Y, Lu J et al. The prognostic value of miR-126 expression in non-small-cell lung cancer: a meta-analysis. Cancer Cell Int. 17, 71 (2017). 
